characiers : it will be capabl: of forming an oxide $\mathrm{RO}_{3}$; its hydride, if it exis's, will be even more unstable than tellurium hydride; its compounds will be easily reduced; it will form definite alloys with metals; and have the specific gravity of about 9.3 . Since then Prof. IV. Preyer, in his "Genetisches System der chemischen Elemente," Berlin, 1893, p. Ioo, among unknown elements, gives one in the sulphur group, thus predicted by Mendeléef. II goes into further details, representing the atomic weight as 213 ; specific gravity, 8.6 ; atomic volume, 247 ; specific heat, 0.03 ; electro-negative, divalent, diamagnetic.

A. Grinwald (B'. 189I, 5, p. 21) appears to have seen the spectrum of this element, whilst observing spectra of tellurium, copper and antimony.

Norweginm is conidered by some chemists to be this missing element; Dr. Preyer is not of that opinion, regarding the claims of norwegium tu be considered an element to be insufficiently supported.

In consideration of Dr. Brauner's work on the atomic weight of tellurium, and the priority of his suggestion of the existence of an accompanying element of higher atomic weight, coupled with his excellent work generally in furthering the claims of the Periodic Law (notably in the cases of beryllium and of the cerium, lanthanum and didymium series), I would suggest that this new element, when isolated from its close union with tellurium, be called Bohemium, in his honour.

C. T. BLANSHARD.

\section{Bright Meteors.}

A PRETTY bright meteor crossed the eastern sky here on Saturday evening last, at about 7.54 p.m. I saw only the flash of light which it cast on the ground and in the sky towards the east, like a momentary weak red flash of lightning. "A shooting-star," said a bystander close 10 me, who saw it fairly well, and who gave me, raughly, this description, ly the stars, of its apparent course: From about R.A. $34^{\circ}$, Decl. $+25^{\circ}$, to about $336^{\circ},+12^{\circ}$. It described this course of $12^{\circ}$ in about a second, and was red in colour, and broke up at last with a red flash, leaving no train of light or of sparks along the tract which it had traversed, so long as for a second or two in which I had time to look towards the direction where he pointed. Tree-:ops intercepted his view beyond the point of this disruption, but the light's sudden extinction there made a much further extension of the track unlikely.

About an hour afterwards, at about 8.49 p.m., I chanced to see another rather fine shooting-star, of about the brightness and colour of the planet Mars at present, de,cending some $16^{\circ}$ or $1 S^{\circ}$ across the northern sky in one and a.half or two seconds, from R.A. $47^{\circ}$, Decl. $+70^{\circ}$, to $100^{\circ}+69^{\circ}$, beginning and disappearing rather suddenly, and of nearly uniformly bright appearance all along its course. It projected no sparks, and, like the earlier meteor, left no train of light or enduring sparks along its track to mark its course.

Prolonged backwards the apparent paths of these two meteors diverge from near a Cassiopeiz, and it may perbaps be that a shower of bright, ruddy meteors from the direction of Cassiopeia was in progress on that date, of which the two meteors here described may have been bright enough members to have been generally noted. The above observations, although those of the lirghter meteor are only of rather slender accuracy, will perhaps be use[ul, in tbat case, to serve for comparison with other records which, at least of the larger meteor of the two, may not imposibly have been noted and presetved elsewhere.

Observatory House, Slough, October I. A. S. HERSCHEL.

\section{Tan-Spots over Dogs' Eyes.}

CAN any of your readers explain the meaning of the tanspots seen so cummonly over the eyes in black-and-tan dogs of most breeds?

When in Melbourne last year, I went carefully over all the dogs in a show, with one of the stewards, and we found the spots in all the black-and-tan terriers, foxhounds, deerhounds, collies, Jurchers, \&-c.; but $I$ could get no information regarding them from the experis.

In some of the highly-bred toy dogs, as the small black-andtan terriers, I found on inquiry that these spots, formenly so

NO. 1 jo2. rOL. 50] very conspicuous, were being bred out, and had nearly dis. appeared. Their persistence through so many strongly-marked varieties, except those of late date, is singular, for there is fairly good proof that when first domesticated the dog was red or bright brown, like the pariah, dingo, \&c.

As far as I can see, we do not find the spots white on a black or dark ground; nor yet black or dark on a white or light ground. My explanation is that they have arisen as a permanent marking after the dogs "sported" to black under domestication, and have been preserved and developed through natural selection. Possibly they are protective, and simulate cyes.

One morning, just at dawn, I had occasion to go out into the garden, and while stooping to examine some flowers, near a fence partly covered with creepers, I sudrienly saw an animal's head looking through, and what seemed to be two black, and secringly large, eyes glared at me. Suspecting that a black leopard was about to spring over, I started back, clapped my hands, and shouted. To my relief, however, I saw a tail wag, and found that the spectator was a coolie's dog I knew very well, and which recognised me. The use of the tanspots-in this case at Jeast-ihen occurred to me.

Nay it not be that the spots thus serve a protective purpose, and have often saved the lives of dogs (black dogs) from their enemies, the smaller felines, such as the clouded leopard, \&c.? Perhaps the matter is not new; but if it is, it seems worth looking into.

I have several dogs about here now with black bodies and heads ; the tan-spots, rather pale, are of the size of a shilling. I have shot one, keeping the skin of the head as a curiosity.

Sibsagar, Asam, September 7.

S. E. PEAL.

\section{Flight of the Albatross.}

At the request of several friends, I enclose, for your inspection, a snap-shot of a northern albatross, which I took en route from Victoria, British Columbia, to San Francisco.

The photograph gives the bird in a position in which the human eye is incapable of seeing it. Strangely enough, the flight of the bird which I photographed had been during the whole course of the morning the subject of much discussion : none of us could imagire bow the force which erabled it to fly at such

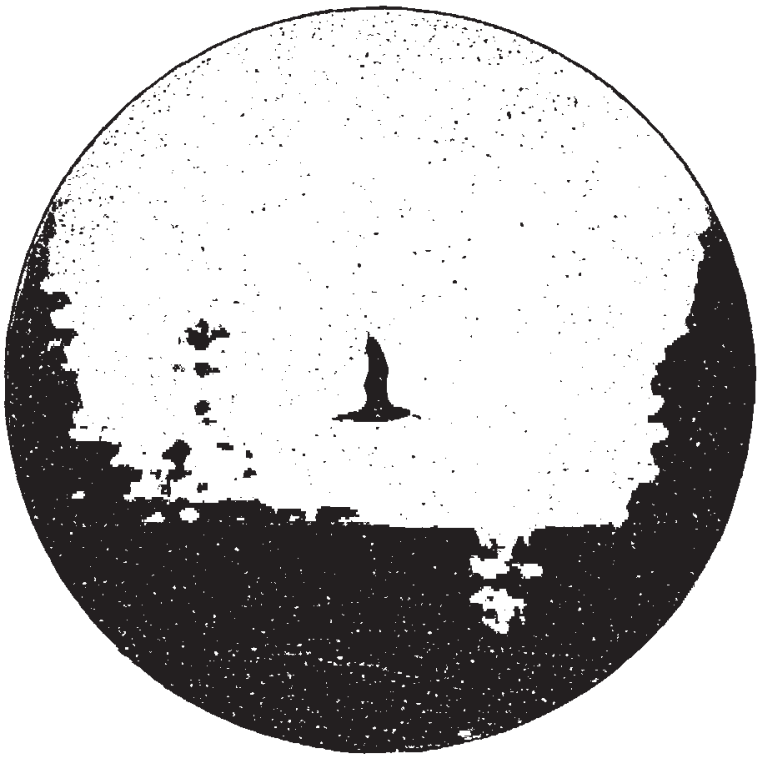

great speed was generated. I remembered having seen some discussion in the papers on the subject, and stated that the matter had been settled to the satisfaction of scientific people, and did my best to explain the theory.

I chose the moment for my photograph when the tird was about fifteen feet from the camera, and sailing alongside of the steamer. The stretch of the wings was estimated by the 
captain of the ship at between eleven and twelve feet, and the indicator on the camera showed these wings apparently at full stretch at the instant that I pressed the button. The result is certainly somewhat astonishing, and I shall be glail to know whether it is worlh comment in your paper; to me it certainly seerns to entirely upset the accepted theories as to the flight of this bird.

stanmore, October 10.

ON THE DOCTRINE OF DISCONTINUITY OF FLUID MOTION, IN CONNECTION WITH THE RESISTANCE AGAINST A SOLID MOVING THROUGH A FLUID.'

III.

§ II. THE accompanying diagram (Fig. I) illustrates the application of the doctrine in question, to a disk kept moving through water or air with a constant velocity, $V$, perpendicular to its own plane. The assumption to which I object as being inconsistent with hydrodynamics, and very far from any
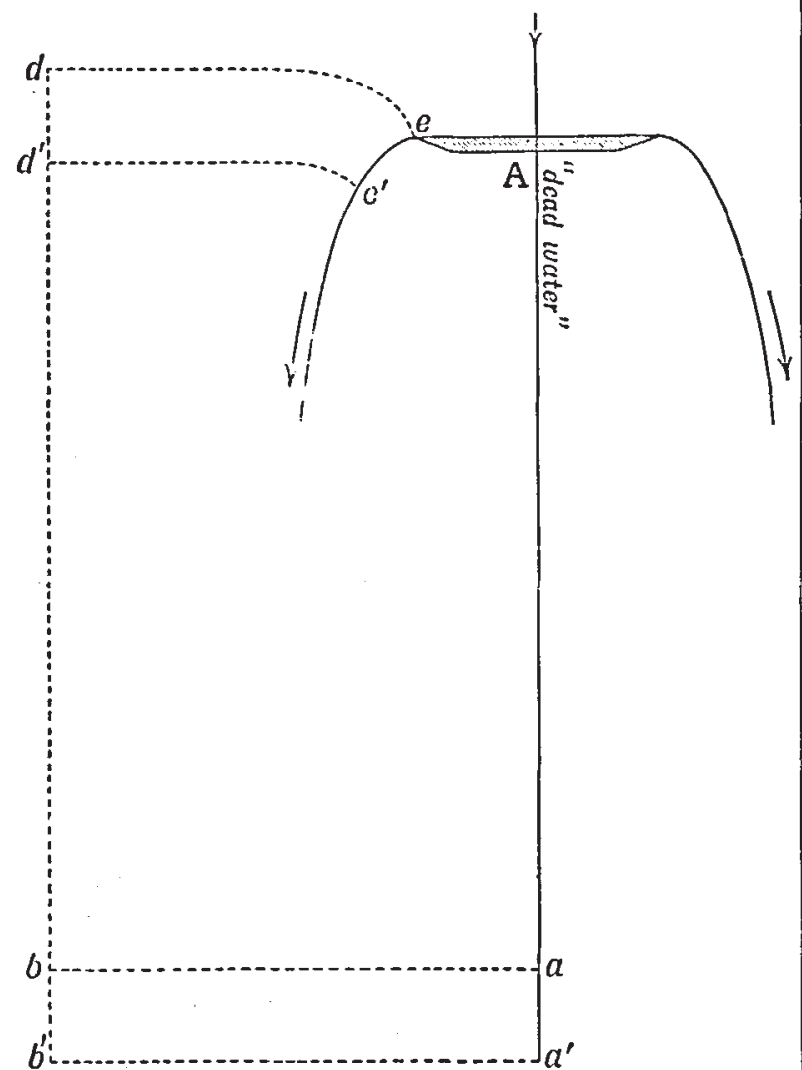

Fig. I.

approximation to the truth for an inviscid incompressible fluid in any circumstances, and utterly at variance with observation of disks or blades (as oar blades) caused to move through water; is, that starting from the edge as represented by the two continuous curves in the diagram, and extending indefinitely rearwards, there is a "surface of discontinuity " on the outside of which the water flows, relatively to the disk, with velocity $V$, and on the inside there is a rear-less mass of "dead water" 2 following close after the disk.

1 Continued from p. 542

2 ' 'his is a technical expression of practical hydraulics, adopted by the English teachers of the dactrine of finite slip between two parss of a homo geneous fluid, to des: gnate water at rest relatively to the dish.

NO. I 302 , vor. 50]
$\S$ 12. The supposed constancy of the velocity on the outside of the supposed surface of discontinuity entails for the inside a constant pressure, and therefore quiescence relatively to the disk, and rearlessness of the "dead water." How such a state of motion could be produced? and what it is in respect to rear? are questions which I may suggest to the teachers of the doctrine, but happily, not going in for an examination in hydrokinetics, I. need not try to answer.

$\S$ I3. But now, supposing the motion of the disk to have been started some finite time, $t$, ago, and consider. ing the consequent necessity $(\$ 9)$ for finiteness of its wake, let $a b, b d$ be lines sufficiently far behind the rear, and beyond one side, of the disturbed water, to pass only through water not sensibly disturbed. We thus have a real finite case of motion to deal with, instead of the inexplicably infinite one of $\S$ II. Let us try if it is possible that for some finite distance from the edge, and from the disk on each side, the motion could be even approximately, if not rigorously, that described in $\$$ II, and indicated by the diagram.

$\S$ I 4 . Let $v$ be the velocity at any point in the axis, $A a$, at distance $y$ from the disk, rearwards. Draw ed perpendicular to the stream lines of the fluid, relatively to the disk supposed at rest.

The "flow" 1 in the line $e d$ is o;

$$
\begin{aligned}
& \text {, " }, d b, \mathrm{~V} \times a b \text {; } \\
& \text { ", ", ba, , o; } \\
& \text { " " }, a A,-\int_{0}^{A a} v d y \text {; } \\
& \text { " " "Ae „, o, by hypothesis. }
\end{aligned}
$$

Hence for the "circulation" 2 in the closed polygon edbaAe, we have

$$
\mathrm{V} \times d b-\int_{0}^{A a} a d y .
$$

Similarly, for the circulation in the same circuit ${ }^{3}$ at a time later by any interval, $\tau$, when the line $b a$ has moved to the position $l^{\prime} a^{\prime}$, and $e d$ to $e^{\prime} d^{\prime}$, we have

$$
\mathrm{V} \times d b-\int_{0}^{A \pi} y^{\prime} d y .
$$

where $v^{\prime}$ denotes, for the later time, $t+\tau$, the velocity in $A a$, at distance $y$ from $A$. Hence the circulation in $e d a A e$ gains in time $r$ an amount equal to

which is the same as

$$
-\int_{0}^{A a}\left(y^{\prime}-v\right) d y
$$

$$
\left.-\int_{0}^{\infty}\left(y^{\prime}-v\right) d\right)^{\prime}
$$

This, by the general theorem of "circulation," must be equal to the gain of circulation in time $\tau$, of all the vortexsheet in its growth from the edge according to the statement of $\S 11$. Hence, with the notation of $\S 10$,

$$
(\Sigma \kappa)^{\prime}-\Sigma \kappa=-\int_{0}^{\infty}\left(v^{\prime}-v\right) d y \text {. }
$$

\& 15. Remarking now that the fluid has only continuous irrotational motion through a finite space all round each of the lines $e d, a b, b a, a \mathrm{~A}$; and all round $A e$ except the space occupied by the disk and the fluid beyond its front side, we have, for the velocity-potential of this motion, relatively to the disk,

$$
v y+\phi(x, y, z, t)
$$

where $\phi$ denotes the velocity-potential of the motion

1 "Vortex Motion" (Thomson), Trans. K.S.E., $1 \& 6 g$.

2 Jbid

3 Kemark that the circulation in $a l b^{\prime} a^{\prime}$ is zero, and therefo:e the circula. tion in $c d b^{\prime} a^{\prime} A$ is equal to that in edlade.

" "Vortex Alotion," I rans. $R$ S.E., refo. 